\title{
Quasi-collinear and partially degenerate four-wave mixing: an alternative explanation of the phase-conjugation property of backward stimulated scattering
}

\author{
Dun Liu
}

Department of Electrical Engineering and Computer Science, University of Michigan, Ann Arbor, Michigan 48109, USA

Guang S. He*)

Photonics Research Laboratory, State University of New York at Buffalo, Buffalo, New York 14260-3000, USA

(Submitted 9 September 1997)

Zh. Éksp. Teor. Fiz. 115, 431-448 (February 1999)

A quasi-collinear and partially degenerate four-wave mixing model is proposed to explain the optical phase-conjugation property of various types of stimulated backscattering.

According to this model, after passing through a phase-disturbed medium or an aberration plate, the input pump beam can be resolved into two portions: a stronger undisturbed regular portion and a weaker phase-disturbed irregular portion. These two portions interfere with each other and create a volume holographic grating in the pumped region of the scattering medium. Only the stronger undisturbed portion of the pump field can excite an initial backward stimulated scattering beam with a regular wavefront. When the latter (as a reading beam) passes through the induced holographic grating, a diffracted wave will be created and then amplified together with the reading beam. A rigorous mathematical analysis shows that under certain conditions the combination of these two portions (the reading wave and the diffracted wave) of the backward stimulated scattering can be an approximate phase-conjugate field of the input pump field. The major theoretical conclusions are basically supported by the experimental results based on a specially designed two-beam interference setup. (C) 1999 American Institute of Physics. [S1063-7761(99)00502-8]

\section{INTRODUCTION}

Optical phase-conjugation is one of the most interesting research subjects in nonlinear optics. ${ }^{1,2}$ So far, there are two major technical approaches to generate optical phaseconjugate waves: one is based on degenerate or partially degenerate four-wave mixing, ${ }^{3-8}$ the other is based on backward stimulated Brillouin, Raman, or Rayleigh-wing scattering. ${ }^{9-14}$ In addition, for certain arrangements, special three-wave mixing, ${ }^{15,16}$ the photon echo technique, ${ }^{17,18}$ and the self-pumped photorefractive effect can also be used to produce phase-conjugate waves. ${ }^{19-21}$

Now backward stimulated scattering (BSS) is one of the most sophisticated techniques to generate optical phaseconjugate waves. A considerable number of theoretical papers on this specific issue have been published since the late 1970s. ${ }^{22-31}$ Most of those theoretical studies have been based on a particular assumption that there is gain discrimination between the phase-conjugate portion and non-phaseconjugate portions of the backward stimulated scattering; only the former can obtain the maximum gain and be effectively amplified. However, for a long time, a clear theoretical model or physical explanation to support this assumption was lacking. Therefore, a better physical understanding of this effect is still necessary, as indicated by Ref. 32. Another feature of most theoretical papers cited here is that both the pump field and the BSS field were represented by a function expressed as an infinite series..$^{9,22,24-31}$ In those cases, it was extremely difficult to obtain a rigorous analytical solution of the wave equation.

In this paper we intend to propose an alternative physical model as well as a novel mathematical approach to explain the phase-conjugation property of BSS. The suggested model is based on a quasi-collinear and partially-degenerate fourwave mixing process, or equivalently, a quasi-collinear holographic wavefront-reconstruction process. The proposed mathematical approach is based on the assumption that both the pump field and the BSS field can be viewed as composed of two portions: a portion of the regular-wave and a portion of the aberration-wave. The advantage of the suggested approach is that an explicit analytic solution of the wave equations can be obtained.

\section{QUASI-COLLINEAR HOLOGRAPHIC INTERACTION MODEL}

Before starting our theoretical discussions, it is helpful to describe briefly the typical experimental setup for demonstrating the phase-conjugation property of the BSS from a given medium. Figure 1a shows the typical experimental setup without using an aberration plate. In this case, a quasiplane pump beam is focused into the center of a scattering medium. Most experimental observations show that the BSS exhibits nearly the same beam size and beam divergence as 


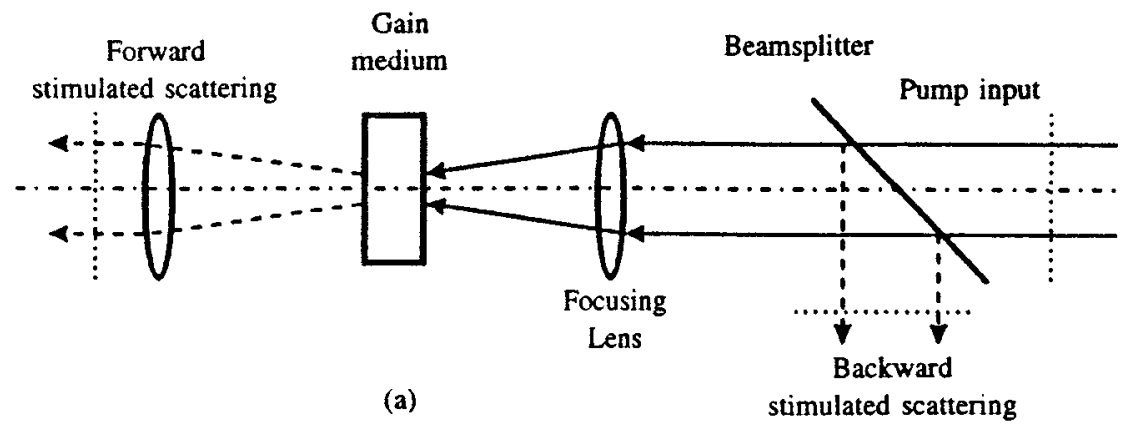

(a)

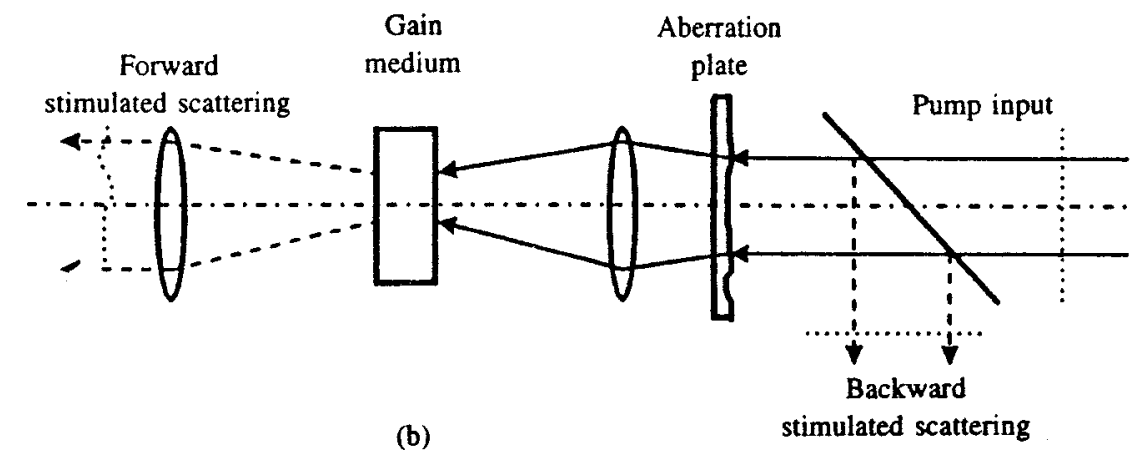

FIG. 1. Experimental setups for observation of a phase-conjugate backward stimulated scattering: (a) without using an aberration plate and (b) with an aberration plate. does the pump beam. These facts can be explained by the following two considerations. First, only those portions of the initial BSS which are propagating within the solid aperture angle (measured from the sample center to the focusing lens) of the pump beam can get the maximum gain length. Second, the divergence angle of the collimated BSS is determined by its spot size in the pumped region of the gain medium, which is limited by the spot size of the pump beam. We can assume that near the focal point region the focused pump beam exhibits a Gaussian transverse intensity distribution. Considering the threshold requirement of the burst of stimulated scattering, the minimum spot size (beam waist) of the stimulated scattering should be slightly smaller or quite closer to that of the pump beam. Therefore, after passing back through the focusing lens the BSS output manifests a slightly smaller or nearly the same beam divergence angle as the pump beam.

Figure $1 \mathrm{~b}$ shows the same experimental setup except that a transparent aberration plate is placed between the beamsplitter and the focusing lens. In this case, after passing back through the aberration plate the BSS may show a significantly reduced aberration influence. That is the typical experimental evidence of the phase-conjugation property of the BSS. $^{10-14}$

To describe how can we employ the quasi-collinear holographic interaction model to explain the basic experimental results mentioned above, it is worth returning to the original idea of Gabor's holography principle. In that case, a coherent light wave passing through a transparent object (phase object), the object is assumed to be such that a considerable part of the wave penetrates undisturbed through it, and a hologram is formed by the interference between the secondary wave arising from the presence of the object and the strong background wave, as clearly described in Ref. 33.
According to this principle, after passing through a phase object the total optical field can be expressed as a superposition of two portions: ${ }^{33}$

$$
\begin{aligned}
U & =U^{(i)}+U^{(s)}=A^{(i)} \exp \left(i \varphi_{i}\right)+A^{(s)} \exp \left(i \varphi_{s}\right) \\
& =\exp \left(i \varphi_{i}\right)\left\{A^{(i)}+A^{(s)} \exp \left[i\left(\varphi_{s}-\varphi_{i}\right)\right]\right\}
\end{aligned}
$$

Here $U^{(i)}$ is the undisturbed part of the transmitted field, $U^{(s)}$ the secondary wave arising from the presence of the object; $A^{(i)}$ and $A^{(s)}$ are their amplitude functions; and $\varphi_{i}$ and $\varphi_{s}$ are the corresponding phase functions, respectively.

The Gabor principle described above is applicable to most phase-conjugation experiments based on BSS. In this case, as shown schematically in Fig. 2, $E(\omega)$ is a quasiplane pump wave; after passing through an aberration plate, the pump field appears as a superposition of two portions: a stronger undisturbed wave $E_{1}(\omega)$ and a weaker distorted wave $E_{2}(\omega)$. After passing through a focusing lens, these two portions interfere with each other in the focal region inside a gain medium and create an induced volume holographic grating that is due to the intensity-dependent refractive index change of the gain medium. Only the $E_{1}(\omega)$ wave is strong enough to fulfill the threshold requirement and to generate an initial BSS wave $E_{3}\left(\omega^{\prime}\right)$, the latter exhibits the same regular wavefront as does the former. When the $E_{3}\left(\omega^{\prime}\right)$ wave passes backward through the induced holographic grating region, a diffracted wave $E_{4}\left(\omega^{\prime}\right)$ is created. Here we see a typical holographic wavefront-reconstruction process: the induced grating is formed by the regular $E_{1}(\omega)$ wave (reference beam) and the irregular $E_{2}(\omega)$ wave (signal beam); the initial backward stimulated scattering $E_{3}\left(\omega^{\prime}\right)$ wave is a reading beam with a regular wavefront like the $E_{1}(\omega)$; therefore, the diffracted wave $E_{4}\left(\omega^{\prime}\right)$, as a recon- 


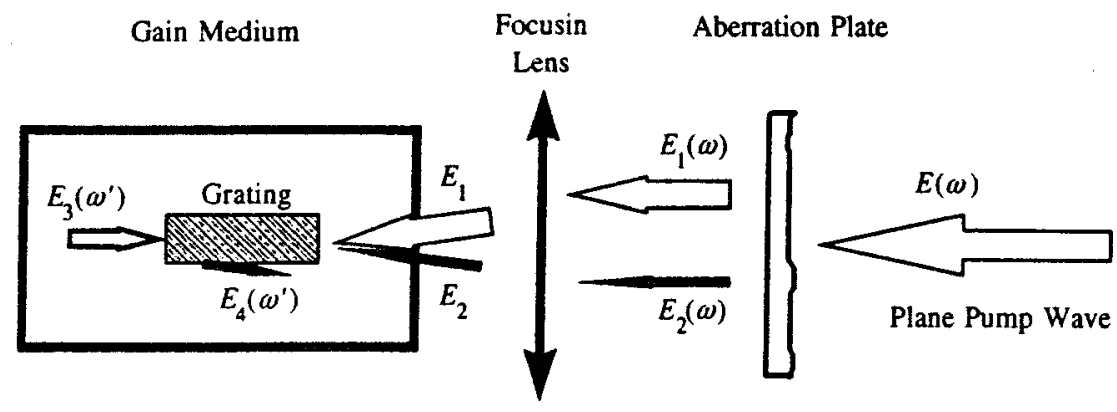

FIG. 2. Schematic illustration of Gabor's holographic model for the phase-conjugation formation of backward stimulated scattering.

structive beam, will be the phase-conjugate replica of the $E_{2}(\omega)$ wave.

Furthermore, the $E_{4}\left(\omega^{\prime}\right)$ wave will experience a further amplification together with the $E_{3}\left(\omega^{\prime}\right)$ wave because both waves have the same signal frequency. In the case of stimulated Brillouin scattering, $\omega \approx \omega^{\prime}$, it is a nearly degenerate quasi-collinear four-wave mixing (FWM) process in the sense of phase-conjugate formation. In the case of stimulated Raman scattering, $\omega>\omega^{\prime}$, there is a partially degenerate and frequency down-converted FWM process. In the case of antiStokes stimulated scattering, $\omega<\omega^{\prime}$, there is a partially degenerate and frequency up-converted FWM process. Based on the explanations described above, one can see that there is a common mechanism (pump field-induced holographic grating) playing the same key role for phase-conjugation formation in both FWM and BSS. This common mechanism is applicable to any types of backward stimulated scattering processes including stimulated Brillouin, Raman, Rayleigh-wing, ${ }^{34,35}$ and Kerr scattering, ${ }^{36-38}$ even though the specific scattering mechanisms are totally different among them.

\section{PUMP FIELD-INDUCED HOLOGRAPHIC GRATING AND STIMULATED SCATTERING GAIN}

Let us consider an isotropic scattering medium. We assume that $\omega$ and $\omega^{\prime}$ are the frequencies of pump wave and stimulated scattering wave, both of which are linearly polarized along the $x$ axis. The induced refractive index change experienced by the stimulated scattering wave can be expressed as

$$
\Delta n\left(\omega^{\prime}\right)=\frac{1}{2 n_{0}\left(\omega^{\prime}\right)} \chi_{e}^{(3)}\left(-\omega^{\prime} ; \omega^{\prime},-\omega, \omega\right)|E(\omega)|^{2},
$$

where $n_{0}\left(\omega^{\prime}\right)$ is the linear refractive index, $\chi_{e}^{(3)}=\chi_{x x x x}^{(3)}$ is a real matrix element of the third-order nonlinear susceptibility tensor, and $E(\omega)$ is the electric field function of the incident pump beam. In the focal region inside the gain medium, the values of local intensity of the pump field, $I(x, y, z, \omega)$ $\propto|E(x, y, z, \omega)|^{2}$, can be quite high with a spatial intensity fluctuation that is due to the interference between the two portions (disturbed and undisturbed) of the pump beam. As a result, an intensity-dependent holographic grating can be formed via the mechanism described by Eq. (2).

If the local pump intensity is high enough, stimulated scattering and subsequent stimulated amplification may occur in the focal region of the pump beam inside the medium. As in the case of one-photon pumped lasing, in steady-state and small-signal approximation the growth of an initial stimulated scattering signal can be described as

$$
I\left(l, \omega^{\prime}\right)=I\left(0, \omega^{\prime}\right) e^{(G-\alpha) l},
$$

where $\omega^{\prime}$ is the frequency of the stimulated Stokes scattering, $I\left(l=0, \omega^{\prime}\right)$ is the initial intensity, $\alpha$ is the linear attenuation coefficient, $G$ is the exponential gain coefficient (in units of $\mathrm{cm}^{-1}$ ), and $l$ is the effective gain length (in units of $\mathrm{cm}$ ) of the medium. The threshold requirement for the burst of an observable stimulated scattering can be written as

$$
e^{(G-\alpha) l} \gg 1
$$

The exponential gain coefficient $G$ is assumed to be proportional to the local intensity of the pump field, i.e.,

$$
G(z)=g I_{0}(z, \omega) .
$$

Here the pump intensity $I_{0}(z, \omega)$ is in units of $\mathrm{MW} / \mathrm{cm}^{2}$, and $g$ is the exponential gain factor in units of $\mathrm{cm} / \mathrm{MW}$.

\section{GENERALIZED DEFINITIONS OF AN OPTICAL PHASE- CONJUGATE WAVE}

The term "optical phase-conjugation" is specially used to described the wavefront reversal property of a backward propagating optical wave with respect to a forward propagating optical wave. Suppose there is an input quasimonochromatic wave with a certain phase-distortion departing from an ideal plane wavefront, i.e.,

$$
\begin{aligned}
E(z, x, y, \omega) & =E(z, x, y) e^{-i \omega t} \\
& =A_{0}(z, x, y) e^{i[k z+\varphi(z, x, y)]} e^{-i \omega t} .
\end{aligned}
$$

Here, $z$ is the longitudinal variable along the propagation direction, $x$ and $y$ are the transverse variables along the bean section, $\omega$ is the frequency of the field, $k=2 \pi n_{0} / \lambda$ is the magnitude of the wave vector; $E(z, x, y)$ is the complex amplitude function, $A_{0}(z, x, y)$ is the real amplitude function, and, $\varphi(z, x, y, k)$ is a phase-distortion function. If there is a backward propagating wave which can be expressed as

$$
\begin{aligned}
E^{\prime}(z, x, y, \omega) & =a E^{*}(z, x, y) e^{-i \omega t} \\
& =a A_{0}(z, x, y) e^{-i[k z+\varphi(z, x, y)]} e^{-i \omega t},
\end{aligned}
$$

where $a$ is any real constant, the field $E^{\prime}(z, x, y, \omega)$ is called the frequency-degenerate phase-conjugate wave of the input field $E(z, x, y, \omega)$. This type of optical phase-conjugate wave can be experimentally generated by using the well-known degenerate four-wave mixing technique. ${ }^{3-7}$ 


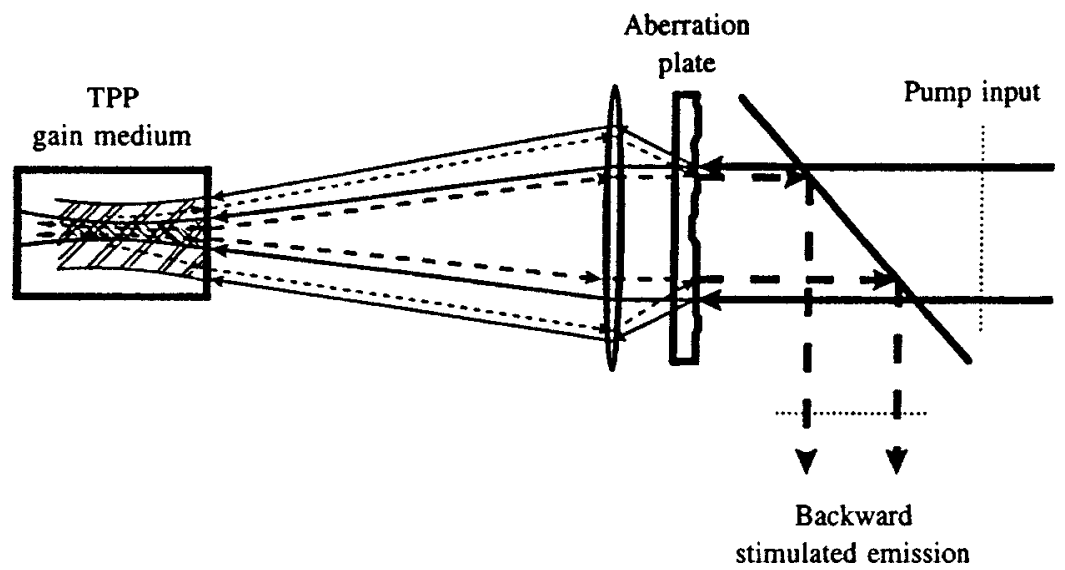

FIG. 3. Schematic illustration of the optical-path geometry for the formation of a phase-conjugate backward stimulated scattering.
In a more general case, if there is a backward propagating optical field with a different frequency $\omega^{\prime}$, which can be written as

$$
E^{\prime \prime}\left(z, x, y, \omega^{\prime}\right)=a A_{0}(z, x, y) e^{-i\left[k^{\prime} z+\varphi(z, x, y)\right]} e^{-i \omega^{\prime} t},
$$

then $E^{\prime \prime}\left(z, x, y, \omega^{\prime}\right)$ can be termed the frequencynondegenerate phase-conjugate wave of the same original field $E(z, x, y, \omega)$. This type of phase-conjugate wave can be experimentally generated by using (i) various BSS with a considerable frequency-shift, ${ }^{12,13}$ or (ii) a partially degenerate FWM. ${ }^{8,39-42}$

\section{DESCRIPTION OF A FOCUSED INPUT PUMP FIELD}

A detailed schematic illustration for the beam-path geometry of the BSS in a scattering medium is shown in Fig. 3. In this case, a quasi-parallel pump beam (thick lines) passes through an aberration plate and is focused into the center of the medium. After passing through the aberration plate, the pump beam can be imagined as composed of two portions. One is a relatively strong undisturbed quasi-plane wave (medium-thick lines); the other is a relatively weak distorted wave (thin lines). These two portions of the input pump beam can interfere with each other in the overlap region and create a volume holographic grating. For the undistorted portion of the input pump beam, the optical field in the focal region can be approximately treated as the fundamental mode of a Hermite-Gaussian beam, i.e., ${ }^{25,43}$

$$
\begin{aligned}
E_{1}(z, x, y, \omega)= & C_{1} \frac{w_{0}}{w(z)} \exp \left\{-\left(x^{2}+y^{2}\right)\right. \\
& \left.\times\left[\frac{1}{w^{2}(z)}-\frac{i k}{2 R(z)}\right]-i \tan ^{-1}\left(\frac{z}{\delta z}\right)\right\} \\
& \times \exp [i(k z-\omega t)]
\end{aligned}
$$

where $C_{1}$ is a real amplitude constant, and the parameters $w$, $R$ and $\delta z$ are determined by

$$
\begin{aligned}
& w^{2}(z)=w_{0}^{2}\left[1+\left(\frac{z}{\delta z}\right)^{2}\right], \quad R(z)=z\left[1+\left(\frac{z}{\delta z}\right)^{2}\right], \\
& \delta z=\frac{k w_{0}^{2}}{2} .
\end{aligned}
$$

Here, $w(z)$ represents the change in the pump-beam size along the $z$ axis near the focal point region, $w_{0}$ is the minimum spot size of the beam at the focal plane, $\delta z$ is the focal depth of the focused beam, and $R(z)$ describes the change of the radius of curvature as a function of $z$. In writing Eq. (9), we simply neglect the amplitude depletion of the pump field within a distance comparable to $\delta z$.

According to Gabor's principle and Eq. (1), the total pump field near the focal point region can be expressed as a combination of two portions:

$$
\begin{aligned}
E(z, x, y, \omega)= & E_{1}(z, x, y, \omega)+E_{2}(z, x, y, \omega) \\
= & {\left[A_{1}(z, x, y)+A_{2}(z, x, y)\right] \exp [i(k z-\omega t)] } \\
= & \left\{C _ { 1 } \frac { w _ { 0 } } { w ( z ) } \operatorname { e x p } \left(-\left(x^{2}+y^{2}\right)\left[\frac{1}{w^{2}(z)}-\frac{i k}{2 R(z)}\right]\right.\right. \\
& \left.\left.-i \tan ^{-1} \frac{z}{\delta z}\right)+C_{2} \exp [i \theta(z, x, y)]\right\} \\
& \times \exp [i(k z-\omega t)] .
\end{aligned}
$$

Here $C_{1}$ and $C_{2}$ are real amplitude constants for normalization, and $\theta(z, x, y)$ is an unknown function depending on the aberration plate. The first term with $C_{1}$ in the braces of Eq. (11) represents the undistorted portion of the pump field; the second term with $C_{2}$ represents the distorted portion of the pump field. It is assumed that the latter amplitude is distributed nearly uniformly near the focal point with a cross section considerably larger than $w_{0}$. As shown in Fig. 3, this assumption is based on the fact that the aberration plate causes a wide and diffuse halo in the focal plane compared to the small focal spot of the undistorted wave. Nevertheless, apart from the focal plane these two portions overlap considerably with each other and can induce a holographic grating.

\section{FORMATION OF PHASE-CONJUGATE WAVE BY BACKWARD STIMULATED SCATTERING}

As shown in Fig. 3, the two portions of the input pump beam can interfere with each other in the overlapping region and create a volume holographic grating. However, on the other hand, since the focused undisturbed portion of the pump beam has a much smaller focal spot size and much higher local intensity than that of the distorted portion, an 
initial BSS (medium-thick dashed lines) with a regular wavefront can be excited only by the stronger undisturbed pump field. In propagating backward this initial BSS experiences further amplification, and creates a diffracted wave through the induced holographic grating. This diffracted portion (thin dashed lines) can be viewed as a secondary seeded signal and therefore will undergo further amplification together with the initial BSS signal. The remaining issue is whether the combination of these two portions of the backward emission can be a phase-conjugate replica of the combination of the two portions of the input pump field.

\subsection{Description of the BSS field: the $E_{1}^{\prime}\left(\omega^{\prime}\right)$ wave}

According to the proposed model, it is assumed that the backward stimulated emission field also consists of two portions and can be expressed as

$$
\begin{aligned}
E^{\prime}\left(z, x, y, \omega^{\prime}\right)= & E_{1}^{\prime}\left(z, x, y, \omega^{\prime}\right)+E_{2}^{\prime}\left(z, x, y, \omega^{\prime}\right) \\
= & {\left[A_{1}^{\prime}(z, x, y)+A_{2}^{\prime}(z, x, y)\right] } \\
& \times \exp \left[i\left(-k^{\prime} z-\omega^{\prime} t\right)\right],
\end{aligned}
$$

where $A_{1}^{\prime}(z, x, y)$ is the complex amplitude function of the initial BSS wave and $A_{2}^{\prime}(z, x, y)$ is the complex amplitude function of the diffracted wave created by the former through the induced holographic grating.

According to the physical model described in Sec. 2, the $A_{1}^{\prime}$ field is generated by the $A_{1}$ field through the BSS process. Therefore, the electric polarization component corresponding to $A_{1}^{\prime}$ field can be formally written as (in SI units)

$$
\begin{aligned}
P_{1}^{\prime}\left(\omega^{\prime}\right)= & i \varepsilon_{0} \chi_{e}^{\prime}\left|A_{1}\right|^{2} A_{1}^{\prime} \exp \left[i\left(-k^{\prime} z-\omega^{\prime} t\right)\right] \\
= & i \varepsilon_{0} \chi_{e}^{\prime} \frac{C_{1}^{2} w_{0}^{2}}{w^{2}(z)} \exp \left[-\frac{2\left(x^{2}+y^{2}\right)}{w^{2}(z)}\right] A_{1}^{\prime} \\
& \times \exp \left[i\left(-k^{\prime} z-\omega^{\prime} t\right)\right],
\end{aligned}
$$

where $\varepsilon_{0}$ is the permittivity of vacuum and $\chi_{e}^{\prime}$ is a phenomenologically introduced effective third-order susceptibility coefficient (a real coefficient) that is employed to describe the gain behavior of the BSS process. The above expression is based on the assumption that the initial BSS experiences an exponential gain, and the exponential gain coefficient is proportional to the intensity of the $A_{1}$ field [see Eq. (5)]. The wave equation of $E_{1}^{\prime}$ can be written as

$$
\nabla^{2} E_{1}^{\prime}-\frac{n_{0}^{2}\left(\omega^{\prime}\right)}{c^{2}} \frac{\partial^{2} E_{1}^{\prime}}{\partial t^{2}}=\mu_{0} \frac{\partial^{2} P_{1}^{\prime}\left(\omega^{\prime}\right)}{\partial t^{2}},
$$

where $\mu_{0}$ is the permeability in vacuum, $c$ the speed of light in vacuum, and $n_{0}\left(\omega^{\prime}\right)$ the linear refractive index at the frequency of $\omega^{\prime}$. In the slowly-varying-amplitude approximation, the solution of Eq. (14) obeys the following equation:

$$
\begin{aligned}
\frac{\partial A_{1}^{\prime}}{\partial z}+\frac{i}{2 k^{\prime}} \nabla_{\perp}^{2} A_{1}^{\prime}= & \frac{g^{\prime} C_{1}^{2}}{2\left[1+(z / \delta z)^{2}\right]} \\
& \times \exp \left[-\frac{2\left(x^{2}+y^{2}\right)}{w^{2}(z)}\right] A_{1}^{\prime},
\end{aligned}
$$

where $g^{\prime}=k^{\prime} \chi_{e}^{\prime}$ is the exponential gain factor of the stimulated emission for a given medium. In order to solve this equation, we could solve for the Fourier transform of $A_{1}^{\prime}$, then obtain the solution of $A_{1}^{\prime}$ through the reverse Fourier transform. ${ }^{25}$ Omitting the intermediate mathematical procedure, the final solution of $A_{1}^{\prime}$ is given by

$$
\begin{aligned}
A_{1}^{\prime}= & C_{1}^{\prime} \frac{w_{0}^{\prime}}{w^{\prime}(z)} \exp \left[\frac{1}{2} g^{\prime} C_{1}^{2}\left(z-z_{0}\right)\right] \exp \left\{-\left(x^{2}+y^{2}\right)\right. \\
& \left.\times\left[\frac{1}{w^{\prime 2}(z)}+\frac{i k^{\prime}}{2 R^{\prime}(z)}\right]+i \tan ^{-1} \frac{z}{\delta z^{\prime}}\right\} .
\end{aligned}
$$

Here $C_{1}^{\prime}$ is a real amplitude constant, the first exponential term represents the amplitude gain effect, the second exponential term describes the behavior of the transverse intensity and wavefront curvature, and $z_{0}$ is the starting position of the initial backward stimulated emission. The other new parameters in the above equation are defined as

$$
\begin{aligned}
& \delta z^{\prime}=\eta \delta z, \quad w_{0}^{\prime 2}=\left(k / k^{\prime}\right) \eta w_{0}^{2} \\
& w^{\prime 2}(z)=w_{0}^{\prime 2}\left[1+\left(\frac{z}{\delta z^{\prime}}\right)^{2}\right] \\
& R^{\prime}(z)=z\left[1+\left(\frac{\delta z^{\prime}}{z}\right)^{2}\right]
\end{aligned}
$$

and

$$
\eta=\frac{1}{3} g^{\prime} C_{1}^{2} \delta z\left[\left(\frac{z}{\delta z}\right)^{3}-\left(\frac{z_{0}}{\delta z}\right)^{3}\right] .
$$

In the condition

$$
\eta=\frac{1}{3} g^{\prime} C_{1}^{2} \delta z\left[\left(\frac{z}{\delta z}\right)^{3}-\left(\frac{z_{0}}{\delta z}\right)^{3}\right] \approx 1,
$$

we have $\delta z^{\prime}=\delta z, w_{0}^{\prime 2} \approx w_{0}^{2}, w^{\prime 2}(z) \approx w^{2}(z)$ and $R^{\prime}(z)$ $=R(z)$. Comparing Eq. (16) to Eq. (11), one can see that only in that case can the $E_{1}^{\prime}$ field be approximately the phaseconjugate replica of the input $E_{1}$ field. Later, we will discuss the real experimental conditions that fulfill the above requirement and will also consider the influence of the difference between $k^{\prime}$ and $k$.

Now let us consider the gain behavior of the backward stimulated emission described by the first exponential term in Eq. (16). We have already chosen $z=0$ at the focal point position in the sample center, and we assume that the initial backward stimulated emission starts roughly from the position $-z_{0} \approx-\delta z / 2$. If the optical path length of the gain medium is much longer than $\delta z$, the effective single-path amplitude gain can be written as

$$
\Delta(d) \approx \exp \left(\frac{1}{2} g^{\prime} C_{1}^{2} \delta z\right) .
$$

If the sample thickness is much shorter than the focal depth, i.e., $d \ll \delta z$, then we have

$$
\Delta(d) \approx \exp \left(\frac{1}{2} g^{\prime} C_{1}^{2} d\right) .
$$


This indicates that the BSS grows exponentially along the whole optical path length within the medium.

\subsection{Description of the BSS field: the $E_{2}^{\prime}$ wave}

Next we shall consider the diffracted $E_{2}^{\prime}$ wave created by the $E_{1}^{\prime}$ wave through the induced holographic grating near the focal region. Like a partly degenerate FWM process, the nonlinear polarization component $P_{2}^{\prime}$, which corresponds to the $E_{2}^{\prime}$ wave, can be written as ${ }^{44,45}$

$$
P_{2}^{\prime}\left(\omega^{\prime}\right)=i \varepsilon_{0} \chi_{e}^{\prime \prime} A_{1} A_{2}^{*} A_{1}^{\prime} \exp \left[i\left(-k^{\prime} z-\omega^{\prime} t\right)\right],
$$

where $\chi_{e}^{\prime \prime}$ is a nominally introduced effective third-order susceptibility (a real coefficient) that is used to characterize the gain behavior of FWM or a grating diffraction process. Assuming that the condition of Eq. (19) is satisfied and the $E_{1}^{\prime}$ field is phase-conjugated with the $E_{1}$ field, then, based on Eqs. (11), (16), and (19), we can obtain an explicit expression for $P_{2}^{\prime}$ :

$$
\begin{aligned}
P_{2}^{\prime}\left(\omega^{\prime}\right)= & i \varepsilon_{0} \chi_{e}^{\prime \prime} \frac{C_{1} C_{2} C_{1}^{\prime} w_{0}^{2}}{w^{2}} \exp \left[-\frac{2\left(x^{2}+y^{2}\right)}{w^{2}(z)}\right] \\
& \times \exp (-i \theta) \exp \left[\frac{1}{2} g^{\prime} C_{1}^{2}\left(z-z_{0}\right)\right] \\
& \times \exp \left[i\left(-k^{\prime} z-\omega^{\prime} t\right)\right] .
\end{aligned}
$$

Substituting Eq. (23) into a nonlinear wave equation like Eq. (14), we find that the complex $A_{2}^{\prime}$ function is governed by the following equation:

$$
\begin{aligned}
\frac{\partial A_{2}^{\prime}}{\partial z}+\frac{i}{2 k^{\prime}} \nabla_{\perp}^{2} A_{2}^{\prime}= & \frac{g^{\prime \prime}}{2} \frac{C_{1} C_{2} C_{1}^{\prime}}{1+(z / \delta z)^{2}} \\
& \times \exp \left[-\frac{2\left(x^{2}+y^{2}\right)}{w^{2}(z)}\right] \exp (-i \theta) \\
& \times \exp \left[\frac{1}{2} g^{\prime} C_{1}^{2}\left(z-z_{0}\right)\right],
\end{aligned}
$$

where $g^{\prime \prime}=k^{\prime} \chi_{e}^{\prime \prime}$. Since $A_{2}^{\prime}$ does not appear on the right-hand side of Eq. (24), we can give a trial solution of $A_{2}^{\prime}$ as

$$
A_{2}^{\prime}=\frac{C_{2} C_{1}^{\prime}}{C_{1}} \exp \left[-\frac{2\left(x^{2}+y^{2}\right)}{w^{2}(z)}\right] A_{2}^{\prime \prime},
$$

where $A_{2}^{\prime \prime}$ is an unknown function to be solved. Substituting Eq. (25) into Eq. (24) leads to

$$
\begin{aligned}
\frac{\partial A_{2}^{\prime \prime}}{\partial z}- & i \frac{2}{\delta z\left[1+(z / \delta z)^{2}\right]}\left(x \frac{\partial A_{2}^{\prime \prime}}{\partial z}+y \frac{\partial A_{2}^{\prime \prime}}{\partial y}\right) \\
& +\frac{i}{2 k^{\prime}}\left(\frac{\partial^{2} A_{2}^{\prime \prime}}{\partial x^{2}}+\frac{\partial^{2} A_{2}^{\prime \prime}}{\partial y^{2}}\right)-\frac{i}{\delta z\left[1+(z / \delta z)^{2}\right]} \\
& \times\left[1-\frac{4\left(x^{2}+y^{2}\right)}{w^{2}(z)}\right] A_{2}^{\prime \prime}+\frac{4\left(x^{2}+y^{2}\right)}{\delta z w^{2}(z)} A_{2}^{\prime \prime} \\
= & \frac{1}{2} g^{\prime \prime} C_{1}^{2} \frac{1}{1+(z / \delta z)^{2}} \exp \left[\frac{1}{2} g^{\prime} C_{1}^{2}\left(z-z_{0}\right)\right] \exp (-i \theta) .
\end{aligned}
$$

This is a rather complicated partial differential equation; however, it can be greatly simplified with the following considerations. First, since there is an exponential term on the right-hand side of Eq. (26), we may expect that the solution of $A_{2}^{\prime \prime}$ should also involve a corresponding exponential gain term. As a result, the condition $\partial A_{2}^{\prime \prime} / \partial z \gg A_{2}^{\prime \prime} / \delta z$ should be fulfilled; therefore, compared to the first term, the fourth and the fifth terms on the left-hand side of Eq. (26) can be neglected. Second, the focal depth is much larger that the beam size, i.e., $\delta z \gg x, y$; the longitudinal variation of the beam intensity is faster than the transverse variation, i.e., $\partial A_{2}^{\prime \prime} / \partial z$ $>\partial A_{2}^{\prime \prime} / \partial x, \partial A_{2}^{\prime \prime} / \partial z>\partial A_{2}^{\prime \prime} / \partial y$. Therefore, the second term on the left-hand side of Eq. (26) can also be neglected. Then Eq. (26) can be finally simplified as

$$
\begin{aligned}
\frac{\partial A_{2}^{\prime \prime}}{\partial z}+\frac{i}{2 k^{\prime}} \nabla_{\perp}^{2} A_{2}^{\prime \prime}= & \frac{g^{\prime \prime} C_{1}^{2}}{2\left[1+(z / \delta z)^{2}\right]} \\
& \times \exp \left[\frac{1}{2} g^{\prime} C_{1}^{2}\left(z-z_{0}\right)\right] \exp (-i \theta) .
\end{aligned}
$$

To solve this equation we can further assume a trial solution,

$$
A_{2}^{\prime \prime}(z, x, y)=A_{2}^{\prime \prime \prime}(z) \exp \left[-i \theta^{\prime}(z, x, y)\right] \text {. }
$$

Here the $A_{2}^{\prime \prime \prime}(z)$ term represents the real amplitude as a function of $z$, and the exponential term represents the phase front as a function of $z, x$, and $y$. Substituting Eq. (28) into Eq. (27), we obtain the following pair of equations:

$$
\begin{aligned}
& \frac{\partial A_{2}^{\prime \prime \prime}}{\partial z}+\frac{A_{2}^{\prime \prime \prime}}{2 k^{\prime}} \nabla_{\perp}^{2} \theta^{\prime}= \frac{g^{\prime \prime} C_{1}^{2}}{2\left[1+(z / \delta z)^{2}\right]} \\
& \times \exp \left[\frac{1}{2} g^{\prime} C_{1}^{2}\left(z-z_{0}\right)\right] \cos \delta \theta, \\
& \frac{\partial \theta^{\prime}}{\partial z}+\frac{1}{2 k^{\prime}}\left[\left(\frac{\partial \theta^{\prime}}{\partial x}\right)^{2}+\left(\frac{\partial \theta^{\prime}}{\partial y}\right)^{2}\right] \\
&=\frac{g^{\prime \prime} C_{1}^{2}}{2\left[1+(z / \delta z)^{2}\right]} \exp \left[\frac{1}{2} g^{\prime} C_{1}^{2}\left(z-z_{0}\right)\right] \frac{\sin \delta \theta}{A_{2}^{\prime \prime \prime}},
\end{aligned}
$$

where

$$
\delta \theta=\theta-\theta^{\prime} .
$$

In the small aberration approximation, the second-order spatial derivative or the square of the first-order spatial derivative of the function $\theta^{\prime}$ can be neglected, then Eqs. (29) can be simplified as

$$
\begin{aligned}
& \frac{\partial A_{2}^{\prime \prime \prime}}{\partial z}=\frac{g^{\prime \prime} C_{1}^{2}}{2\left[1+(z / \delta z)^{2}\right]} \exp \left[\frac{1}{2} g^{\prime} C_{1}^{2}\left(z-z_{0}\right)\right] \cos \delta \theta, \\
& \frac{\partial \theta^{\prime}}{\partial z}=\frac{g^{\prime \prime} C_{1}^{2}}{2\left[1+(z / \delta z)^{2}\right]} \exp \left[\frac{1}{2} g^{\prime} C_{1}^{2}\left(z-z_{0}\right)\right] \frac{\sin \delta \theta}{A_{2}^{\prime \prime \prime}} .
\end{aligned}
$$

As mentioned in Sec. 5, the distorted portion of the input pump field has a uniform amplitude distribution near the focal point region [see Eq. (11)]. This assumption is based on the fact that the beam waist of this portion is considerably larger than that of the undistorted portion of the pump field (see Fig. 3). In Fig. 3 one can also see that for the distorted portion of the pump field, the variation of the shape of the 
beam waist along the $z$ axis is negligible within the focal depth range. Hence we can further assume that the longitudinal variation of the wavefront of the distorted portion of the pump field within the focal depth range can be neglected, i.e.,

$$
\partial \theta / \partial z \approx 0 .
$$

Subtracting the second equation of Eq. (30) from Eq. (31), we obtain a new pair of coupled equations as

$$
\begin{aligned}
& \frac{\partial A_{2}^{\prime \prime \prime}}{\partial z}=\frac{g^{\prime \prime} C_{1}^{2}}{2\left[1+(z / \delta z)^{2}\right]} \exp \left[\frac{1}{2} g^{\prime} C_{1}^{2}\left(z-z_{0}\right)\right] \cos \delta \theta, \\
& \frac{\partial(\delta \theta)}{\partial z}=-\frac{g^{\prime \prime} C_{1}^{2}}{2\left[1+(z / \delta z)^{2}\right]} \exp \left[\frac{1}{2} g^{\prime} C_{1}^{2}\left(z-z_{0}\right)\right] \frac{\sin \delta \theta}{A_{2}^{\prime \prime \prime}} .
\end{aligned}
$$

Dividing the first equation by the second in Eqs. (32), we find

$$
\frac{\partial A_{2}^{\prime \prime \prime}}{\partial(\delta \theta)}=-A_{2}^{\prime \prime \prime} \frac{\cos \delta \theta}{\sin \delta \theta} .
$$

This implies that

$$
A_{2}^{\prime \prime \prime}(z) \sin \delta \theta(z)=A_{2}^{\prime \prime \prime}\left(-z_{0}\right) \sin \delta \theta\left(-z_{0}\right)=B,
$$

where $B$ is a constant considerably smaller than $A_{2}^{\prime \prime \prime}$. Substituting $\cos \delta \theta=\sqrt{\left(A_{2}^{\prime \prime \prime}\right)^{2}-B^{2}} / A_{2}^{\prime \prime \prime}$ into the first equation of Eqs. (32), we have

$$
\begin{aligned}
\frac{A_{2}^{\prime \prime \prime}}{\sqrt{\left(A_{2}^{\prime \prime \prime}\right)^{2}-B^{2}}} \frac{\partial A_{2}^{\prime \prime \prime}}{\partial z}= & \frac{g^{\prime \prime} C_{1}^{2}}{2\left[1+(z / \delta z)^{2}\right]} \\
& \times \exp \left[\frac{1}{2} g^{\prime} C_{1}^{2}\left(z-z_{0}\right)\right] .
\end{aligned}
$$

Since $B^{2} \ll\left(A_{2}^{\prime \prime \prime}\right)^{2}$, Eq. (35) can be approximately rewritten as

$$
\frac{\partial A_{2}^{\prime \prime \prime}}{\partial z}=\frac{g^{\prime \prime} C_{1}^{2}}{2\left[1+(z / \delta z)^{2}\right]} \exp \left[\frac{1}{2} g^{\prime} C_{1}^{2}\left(z-z_{0}\right)\right] .
$$

Now let us consider the physical meaning of the factors $g^{\prime} C_{1}^{2}$ and $g^{\prime \prime} C_{1}^{2}$. The former is used to describe the gain of the $A_{1}^{\prime}$ field due to stimulated scattering amplification, the latter is nominally employed to describe the growth of the $A_{2}^{\prime}$ field through the holographic grating diffraction (or the equivalent four-wave mixing). It is important to point out that the initial $A_{2}^{\prime}$ field can be viewed as a secondary seed signal, which will experience an additional gain from the stimulated scattering amplification like that of the $A_{1}^{\prime}$ field. Under most BSS experimental conditions, the subsequent gain of the $A_{2}^{\prime}$ field comes mainly from the stimulated amplification rather than the equivalent FWM or the grating diffraction. Hence, in Eq. (36) we can replace the $g^{\prime \prime} C_{1}^{2}$ term by the $g^{\prime} C_{1}^{2}$ term, and rewrite Eq. (36) as

$$
\frac{\partial A_{2}^{\prime \prime \prime}}{\partial z}=\frac{g^{\prime} C_{1}^{2}}{2\left[1+(z / \delta z)^{2}\right]} \exp \left[\frac{1}{2} g^{\prime} C_{1}^{2}\left(z-z_{0}\right)\right] .
$$

The final solution of this equation is

$$
A_{2}^{\prime \prime \prime}=\exp \left[\frac{1}{2} g^{\prime} C_{1}^{2}\left(z-z_{0}\right)\right] \text {. }
$$

To obtain this solution, we have assumed that $1 / \sqrt{1+(z / \delta z)^{2}} \approx 1$, which is valid for $z<\delta z$. From Eq. (38) one can find the initial value of $A_{2}^{\prime \prime \prime}$ at $z=-z_{0}$

$$
A_{2}^{\prime \prime \prime}\left(-z_{0}\right)=1 \text {. }
$$

Next, the remaining issue is to consider the phase function $\theta^{\prime}(z, x, y)$ of the $A_{2}^{\prime \prime}$ field expressed by Eq. (28). From Eq. (34) we have

$$
\sin \delta \theta(z)=\sin \delta \theta\left(-z_{0}\right) \frac{A_{2}^{\prime \prime \prime}\left(-z_{0}\right)}{A_{2}^{\prime \prime \prime}(z)} .
$$

Substituting Eqs. (38) and (39) into Eq. (40) leads to

$$
\begin{aligned}
\sin \delta \theta(z)= & \sin \left[\theta(z)-\theta^{\prime}(z)\right]=\sin \delta \theta\left(-z_{0}\right) \\
& \times \exp \left[-\frac{1}{2} g^{\prime} C_{1}^{2}\left(z-z_{0}\right)\right] .
\end{aligned}
$$

From Eq. (41) we see that during backward propagation the phase front of the $A_{2}^{\prime \prime}$ field is getting closer and closer to the phase front of the $A_{2}$ field. Specifically, if the stimulated gain is high enough, i.e.,

$$
\frac{1}{2} g^{\prime} C_{1}^{2}\left(z-z_{0}\right) \gg 1
$$

we find

$$
\sin \left[\theta(z)-\theta^{\prime}(z)\right] \rightarrow 0, \quad \theta^{\prime}(z) \rightarrow \theta(z) .
$$

Based on Eqs. (25), (28), (38) and (43), the diffracted portion of the backward stimulated scattering field can be finally obtained as

$$
\begin{aligned}
A_{2}^{\prime}= & \frac{C_{2} C_{1}^{\prime}}{C_{1}} \exp \left[\frac{1}{2} g^{\prime} C_{1}^{2}\left(z-z_{0}\right)\right] \\
& \times \exp \left[-\frac{2\left(x^{2}+y^{2}\right)}{w^{2}(z)}\right] \exp (-i \theta) .
\end{aligned}
$$

\subsection{The total BSS field: the $E_{1}^{\prime}+E_{2}^{\prime}$ wave}

Based on Eqs. (12), (16) and (44), the total BSS field can finally be written as

$$
\begin{aligned}
E^{\prime}(z, x, y, \omega)= & {\left[A_{1}^{\prime}(z, x, y)+A_{2}^{\prime}(z, x, y)\right] \exp \left[i\left(-k^{\prime} z-\omega^{\prime} t\right)\right] } \\
= & \frac{C_{1}^{\prime}}{C_{1}} \exp \left[\frac{1}{2} g^{\prime} C_{1}^{2}\left(z-z_{0}\right)\right]\left\{C _ { 1 } \frac { w _ { 0 } } { w ( z ) } \operatorname { e x p } \left(-\left(x^{2}\right.\right.\right. \\
& \left.\left.+y^{2}\right)\left[\frac{1}{w^{2}(z)}+\frac{i k^{\prime}}{2 R(z)}\right]+i \tan ^{-1} \frac{z}{\delta z}\right) \\
& \left.+C_{2} \exp \left[-\frac{2\left(x^{2}+y^{2}\right)}{w^{2}(z)}\right] \exp (-i \theta)\right\} \\
& \times \exp \left[i\left(-k^{\prime} z-\omega^{\prime} t\right)\right] .
\end{aligned}
$$

The above expression is obtained assumming that the requirements described by Eqs. (19) and (42) are fulfilled. Here we can combine these two requirements as

$$
\eta=\frac{1}{3} g^{\prime} C_{1}^{2} \delta z\left[\left(\frac{z}{\delta z}\right)^{3}-\left(\frac{z_{0}}{\delta z}\right)^{3}\right] \approx 1,
$$




$$
\frac{1}{2} g^{\prime} C_{1}^{2}\left(z-z_{0}\right) \gg 1
$$

On the other hand, the total input pump field is given by Eq. (11) and can be rewritten as

$$
\begin{aligned}
E(z, x, y, \omega)= & {\left[A_{1}(z, x, y)+A_{2}(z, x, y)\right] \exp [i(k z-\omega t)] } \\
= & \left\{C _ { 1 } \frac { w _ { 0 } } { w ( z ) } \operatorname { e x p } \left(-\left(x^{2}+y^{2}\right)\left[\frac{1}{w^{2}(z)}\right.\right.\right. \\
& \left.\left.-\frac{i k}{2 R(z)}\right]-i \tan ^{-1} \frac{z}{\delta z}\right) \\
& \left.+C_{2} \exp (i \theta)\right\} \exp [i(k z-\omega t)] .
\end{aligned}
$$

Comparing Eq. (45) to Eq. (47), if we neglect the difference between $k$ and $k^{\prime}$ and only consider the central portion of the field within the region of

$$
\left[2\left(x^{2}+y^{2}\right) / w^{2}(z)\right]<1,
$$

we have

$\left[A_{1}^{\prime}(z, x, y)+A_{2}^{\prime}(z, x, y)\right] \propto\left[A_{1}(z, x, y)+A_{2}(z, x, y)\right]^{*}$.

Based on the above relationship, one can conclude that the total BSS field can be approximately phase-conjugate to the total input pump field provided that certain conditions hold.

\section{DISCUSSIONS}

The conclusion that a BSS field can be an approximate phase-conjugate of the input pump field holds under certain conditions. First, the gain requirements expressed by Eq. (46) should be fulfilled. Assuming that the effective gain length of the nonlinear medium is nearly determined by the focal depth of the focused pump beam, e.g., $z$ $=(0.4-0.5) \delta z$ and $z_{0}=-(0.4-0.5) \delta z$, according to the first requirement expressed by Eq. (46)

$$
g^{\prime} C_{1}^{2} \delta z \approx 12-23,
$$

the required gain of the intensity of a small BSS signal should be

$$
\begin{aligned}
\exp \left[g^{\prime} C_{1}^{2}\left(z-z_{0}\right)\right] & \approx \exp \left(g^{\prime} C_{1}^{2} \delta z\right) \approx \exp (12-23) \\
& \approx 1 \times 10^{5}-1 \times 10^{10}
\end{aligned}
$$

In this case, the second requirement of Eq. (46) is automatically fulfilled. The high gain requirement can be readily fulfilled in most experimental conditions for observing the backward stimulated scattering without using any optical feedback devices. For example, the values of the exponential gain factor $g_{B}$ of stimulated Brillouin scattering for the common transparent solvents (such as acetone, benzene, and toluene) are estimated to be $0.01-0.03 \mathrm{~cm} / \mathrm{MW} \cdot{ }^{32,44}$ Assuming the typical pump intensity is $I_{0} \simeq 500 \mathrm{MW} / \mathrm{cm}^{2}$ and the effective gain length (focal depth) is $\delta z \simeq 1.5 \mathrm{~cm}$, the gain should be

$$
g_{B} I_{0} \delta z \approx 8-22 .
$$

Compared Eq. (52) to Eq. (51) we see that the requirements for observing the phase-conjugate property of a BSS wave can be basically fulfilled by common experimental conditions.

The phase-conjugation property of the BSS from a gain medium, in general, is not perfect for the following reasons. First, all the mathematical derivations described above are based on the small-aberration approximation, so that a substantial part of the distorted pump wave may overlap the undisturbed pump wave in the focal region to generate a holographic grating. Second, only the major (central) part of the BSS may exhibit high-fidelity phase-conjugation, as indicated by the requirement of Eq. (48). One can expect that under a larger aberration influence, the fidelity of phaseconjugation behavior should become poorer.

So far the difference between $k$ and $k^{\prime}$ has been ignored, which has a certain influence on the fidelity of the phaseconjugation of a BSS wave. This influence should be essentially the same as when we create a hologram by using two beams of wavelength $\lambda$ and then read this hologram by using another beam of wavelength $\lambda^{\prime}$. Nevertheless, comparing Eq. (45) to Eq. (47) one can see that the difference between $k$ and $k^{\prime}$ has no influence on the relationship $\theta^{\prime}(z)=\theta(z)$, which is the most essential requirement for distortion compensation. But the difference between $k$ and $k^{\prime}$ does affect the radius of curvature of the undistorted part of a BSS field and causes an apparent displacement of the focal point of the BSS wave.

It should also be noted that the holographic model employed in this work is qualitatively compatible with the existing theoretical explanation for phase-conjugate formation of backward stimulated Brillouin scattering, which is based on the assumption that there is a highly nonuniform pump intensity distribution, i.e., a volume speckle pattern in the focal region. Only the phase-conjugate portion of the BSS field whose intensity distribution best matches the nonuniform gain distribution, experiences the maximum exponential gain coefficient, twice that of the non-phase-conjugate portion. $^{24,44}$ In terms of the holographic model the volume speckle is a result of interference between the undistorted and distorted parts of the pump field.

Finally, it is important to indicate that the Gabor holographic interaction model and the theoretical treatment presented in this paper are suitable not only for various BSS processes but also for other kinds of backward coherent emission processes, provided that there is a high exponential gain mechanism for small initial backward coherent signal as well as an effective holographic grating induced by the pump field. ${ }^{46}$ Recently, we have observed a nearly perfect phaseconjugation property in the backward frequency-upconverted stimulated emission from a two-photon pumped lasing medium. $^{47}$ This observation may suggest a new technical approach to generate optical phase-conjugate waves and can be explained very well based on the same theoretical model described here. ${ }^{48-50}$

\section{SOME RECENT EXPERIMENTAL RESULTS}

So far, most experimental studies of phase-conjugation fidelity of BSS have been based on measurements of the 


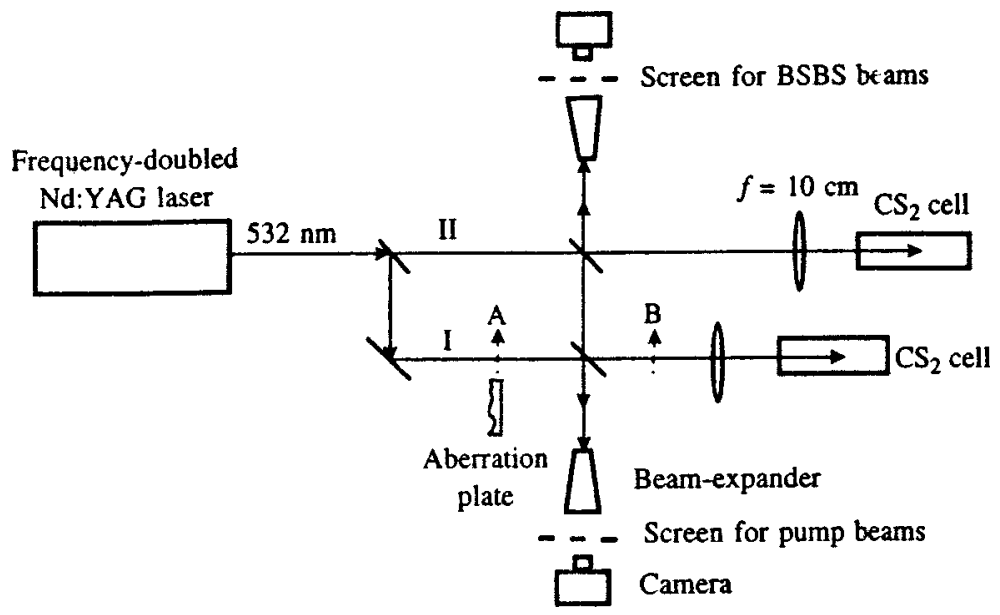

FIG. 4. Experimental setup for measuring the phaseconjugation property of the backward stimulated Brillouin scattering with two-beam interference method.

near-field and far-field distributions. We present here some recent experimental results on the phase-conjugation property of backward stimulated Brillouin scattering (BSBS), based on measuring the fidelity of wavefront reconstruction by using the two-beam interference technique. The advantage of this method is that it can provide both near-field and the wavefront information of the tested beams.

The experimental setup is shown schematically in Fig. 4. A 532-nm master pump laser beam was provided by a $Q$-switched and frequency-doubled pulsed $\mathrm{Nd}$ :YAG laser source; the pulse duration, beam size and divergence angle of this beam were $10 \mathrm{~ns}, 4 \mathrm{~mm}$, and $1 \mathrm{mrad}$, respectively. After passing through a beamsplitter and a reflecting mirror, that master beam was divided into two beams, which were finally focused into two $10-\mathrm{cm}$-long $\mathrm{CS}_{2}$ liquid cells through two $f=10 \mathrm{~cm}$ focusing lenses. The intensities of these two pump beams could be adjusted separately and were high enough to generate BSBS in both liquid cells. Furthermore, by means of two-edge beamsplitters and a $10 \times$ beam expander, the interference pattern of the two incident pump beams could be observed on a screen and recorded by a camera. In the same manner, the interference pattern of the two BSBS beams from these two liquid cells could be also observed in another screen. In order to test the wavefrontreconstruction ability, a hydrofluoric acid-etched glass slide was used as an aberration plate, which could introduce an aberration of 10-15 mrad on the pump beam (I). This aberration plate can be placed either at position A or position $B$.

Figure 5a shows the photograph of the pump beam II: here we see a relatively uniform intensity distribution in the beam section. Figure $5 b$ shows the photograph of the pump beam I after passing through an aberration plate placed at position $\mathrm{A}$; here we see a random transverse intensity fluctuation caused by the aberration plate. Figure $5 \mathrm{c}$ shows the
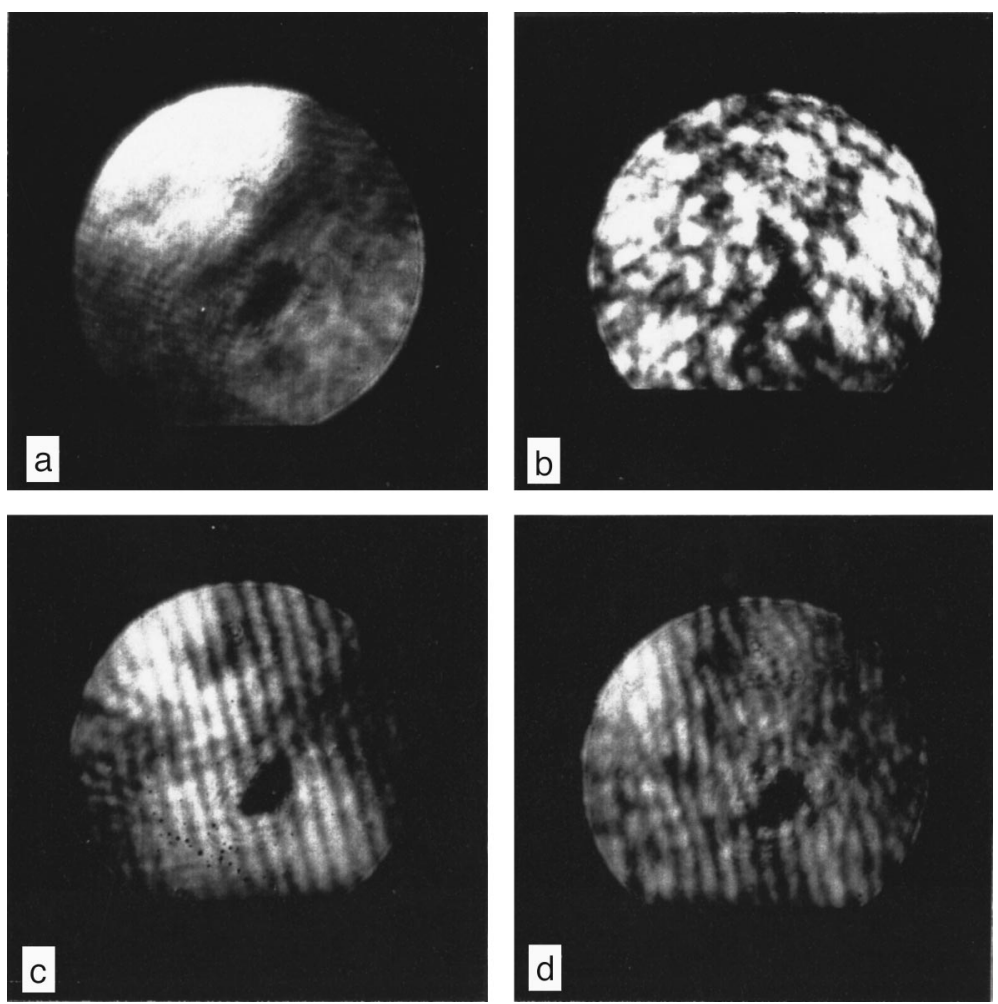

FIG. 5. Photographs of (a) the pump beam II, (b) the pump beam I after passing through an aberration plate, (c) the interference pattern of the two pump beams without no aberration plate, and (d) the interference pattern of the two pump beams with aberration plate in position A shown in Fig. 4. 

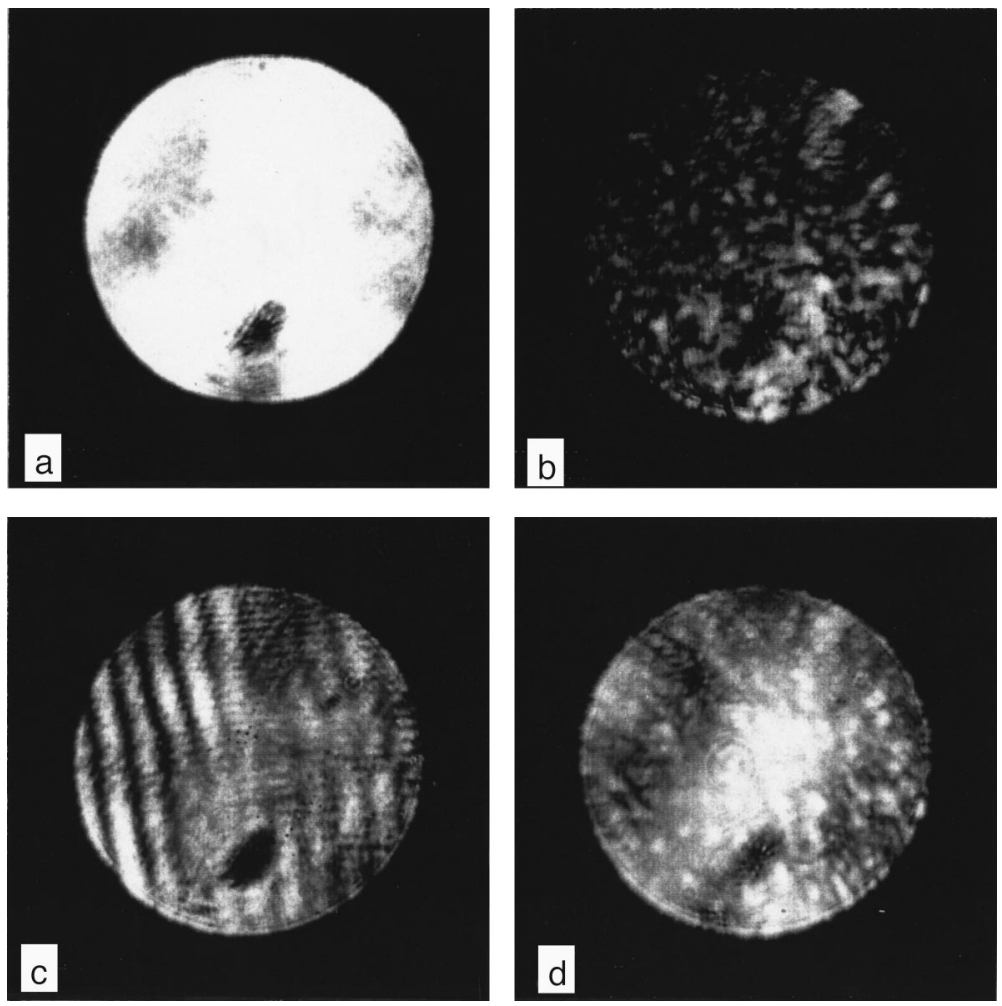

FIG. 6. Photographs of (a) the BSBS beam excited by the pump beam II, (b) the BSBS beam excited by the pump beam I passing through an aberration plate, (c) the interference pattern of the two BSBS beams with no aberration plate, and (d) the interference pattern of the two BSBS beams with an aberration plate in position B shown in Fig. 4. photograph of the interference pattern formed by the two incident pump beams with no aberration plate; here we can see the regular and straight fringes that indicate a nearly ideal plane wavefront for both beams. Finally, Fig. 5d shows a photograph of the interference pattern when the aberration plate was placed at position A; here we can no longer see any clear and regular fringes because of the severe aberration influence on one beam. To obtain the photographs shown in Fig. $5 \mathrm{c}$ and $5 \mathrm{~d}$, the intensities of the two interfering beams were kept nearly the same.

Under the same conditions, a set of photographs can be obtained for the BSBS beams from the two $\mathrm{CS}_{2}$ liquid cells. Figure 6 a shows the photograph of the BSBS beam excited by the pump beam II: here we see a relatively uniform intensity distribution with no discrete-spot structure. Figure $6 \mathrm{~b}$ shows a photograph of the BSBS beam excited by the pump beam I with the aberration plate at position A. Here we see a randomly fluctuating intensity distribution, analogous to that shown in Fig. 5b. Figure 6c shows a photograph of the regular interference pattern formed by two BSBS beams with no aberration plate. Compared Fig. 6c to Fig. 5c, one can find that the two stimulated backscatter beams exhibit a nearly ideal plane wavefront, with no aberration plate. Finally, Fig. $6 \mathrm{~d}$ shows a photograph of the interference pattern formed by the two BSBS beams with the aberration plate at position B. In this case we can still see the clear fringes, although there is a small irregularity among them in comparison with Fig. $6 c$.

The results shown in Fig. $6 \mathrm{c}$ and $6 \mathrm{~d}$ can be readily explained in terms of the two basic theoretical conclusions given in Sec. 7. The first conclusion is that if the stimulated scattering gain is high enough and the aberration influence is small, one can expect a nearly perfect wavefront reconstruc- tion. The result shown in Fig. 6c basically supports this conclusion. The second conclusion is that under a large aberration influence, the wavefront reconstruction will not be perfect. The result shown in Fig. 6d basically supports that conclusion. In the latter case, when the BSBS beam from the second liquid cell passed through the aberration plate at position $\mathrm{B}$, the original wavefront of the pump beam I can be essentially, but not perfectly, reconstructed.

${ }^{*}$ E-mail: gshe@acsu.buffalo.edu

${ }^{1}$ Optical Phase Conjugation, ed. by R. A. Fisher, Academic Press, New York (1983).

${ }^{2}$ A. Yariv, IEEE J. Quantum Electron. 14, 650 (1978).

${ }^{3}$ R. W. Hellwarth, J. Opt. Soc. Am. 67, 1 (1977).

${ }^{4}$ D. M. Bloom and G. C. Bjorklund, Appl. Phys. Lett. 31, 592 (1977).

${ }^{5}$ A. Yariv and D. M. Pepper, Opt. Lett. 1, 16 (1977).

${ }^{6}$ D. G. Steel, R. C. Lind, J. F. Lam, and C. R. Giuliano, Appl. Phys. Lett. 35, 376 (1979).

${ }^{7}$ R. L. Abrams and R. C. Lind, Opt. Lett. 2, 94 (1978); 3, 205 (1978).

${ }^{8}$ G. Martin and R. W. Hellwarth, Appl. Phys. Lett. 34, 371 (1979).

${ }^{9}$ B. Ya. Zel'dovich, V. I. Popovichev, V. V. Ragul'skii, and F. S. Faizullov, JETP Lett. 15, 109 (1972).

${ }^{10}$ O. Yu. Nosach, V. I. Popovichev, V. V. Ragul'skii, and F. S. Faizullov, JETP Lett. 16, 435 (1972).

${ }^{11}$ V. Wang and C. R. Giuliano, Opt. Lett. 2, 4 (1978).

${ }^{12}$ A. I. Sokolovskaya, G. L. Brekhovskikh, and A. D. Kudryavtseva, Opt. Commun. 24, 74 (1978).

${ }^{13}$ A. D. Kudriavtseva, A. I. Sokolovskaia, J. Gazengel, N. P. Xuan, and G. Rivoire, Opt. Commun. 26, 446 (1978).

${ }^{14}$ E. J. Miller, M. S. Malcuit, and R. W. Boyd, Opt. Lett. 15, 1188 (1990).

${ }^{15}$ A. Yariv, Opt. Commun. 21, 49 (1977).

${ }^{16}$ P. V. Avizonis, F. A. Hopf, W. D. Bamberger, S. F. Jacobs, A. Tomita, and K. H. Womack, Appl. Phys. Lett. 31, 435 (1977).

${ }^{17}$ N. C. Griffen and C. V. Heer, Appl. Phys. Lett. 33, 865 (1978).

${ }^{18}$ M. Fujita, H. Nakasuka, H. Nakanishi, and M. Matsuoka, Phys. Rev. Lett. 42, 974 (1979). 
${ }^{19}$ J. O. White, M. Cronin-Golomb, B. Fischer, and A. Yariv, Appl. Phys. Lett. 40, 450 (1982).

${ }^{20}$ J. Feinberg, Opt. Lett. 7, 486 (1982).

${ }^{21}$ M. Cronin-Golomb, B. Fischer, J. O. White, and A. Yariv, Appl. Phys. Lett. 42, 919 (1983).

${ }^{22}$ R. W. Hellwarth, J. Opt. Soc. Am. 68, 1050 (1978).

${ }^{23}$ H. Hsu, Appl. Phys. Lett. 34, 855 (1979).

${ }^{24}$ B. Ya. Zel'dovich, N. F. Pilipetsky, and V. V. Shkunov, Principles of Phase Conjugation, Springer-Verlag, Berlin (1985).

${ }^{25}$ G. G. Kochemasov and V. D. Nikolaev, Kvant. Elektron. (Moscow) 4, 115 (1977) [Sov. J. Quantum Electron. 7, 60 (1977)].

${ }^{26}$ I. M. Bel'dyugin, M. G. Galushkin, E. M. Zemskov, and V. I. Mandrosov, Kvant. Elektron. (Moscow) 3, 734 (1976) [Sov. J. Quantum Electron. 6, 1349 (1976)].

${ }^{27}$ B. Ya. Zel'dovich and V. V. Shkunov, Kvant. Elektron. (Moscow) 4, 1090 (1977) [Sov. J. Quantum Electron. 7, 610 (1977)].

${ }^{28}$ N. B. Baranova, B. Ya. Zel'dovich, and V. V. Shkunov, Kvant. Elektron. (Moscow) 5, 973 (1978) [Sov. J. Quantum Electron. 8, 559 (1978)].

${ }^{29}$ N. B. Baranova and B. Ya. Zel'dovich, Kvant. Elektron. (Moscow) 7, 973 (1980) [Sov. J. Quantum Electron. 10, 555 (1980)].

${ }^{30}$ V. I. Bespalov, V. G. Manishin, and G. A. Pasmanik, Zh. Éksp. Teor. Fiz. 77, 1756 (1979) [Sov. Phys. JETP 50, 879 (1979)].

${ }^{31}$ V. G. Sidorovich and V. V. Shkunov, Zh. Tekh. Fiz. 49, 816 (1979) [Sov. Phys. Tech. Phys. 24, 472 (1979)].

${ }^{32}$ Y. R. Shen, The Principles of Nonlinear Optics, Wiley, New York (1984), pp. $189,254$.

${ }^{33}$ M. Born and E. Wolf, Principles of Optics, 6-th ed., Pergamon, Oxford (1983), p. 453.

${ }^{34}$ D. I. Mash, V. V. Morozov, V. S. Starunov, and I. L. Fabelinskii, JETP Lett. 2, 25 (1965).
${ }^{35}$ G. I. Zaitsev, Y. I. Kyzylasov, V. S. Serarunov, and I. L. Fabelinskii, JETP Lett. 6, 35 (1967)

${ }^{36}$ G. S. He and P. N. Prasad, Phys. Rev. A 41, 2687 (1990).

${ }^{37}$ G. S. He, R. Burzynski, and P. N. Prasad, J. Chem. Phys. 93, 7647 (1990).

${ }^{38}$ G. S. He and G. C. Xu, IEEE J. Quantum Electron. 28, 323 (1992).

${ }^{39}$ J. Nilsen and A. Yariv, Opt. Commun. 39, 199 (1981).

${ }^{40}$ V. M. Arutunyan, G. G. Adonts, A. R. Aramyan, S. P. Ishkhanyan, E. G. Kanetsyan, T. A. Papasyan, and S. M. Sarkisyan, Opt. Appl. 13, 347 (1983).

${ }^{41}$ H. A. Mackenzie, D. J. Hagan, and H. A. Al-Attar, IEEE J. Quantum Electron. QE-22, 1328 (1986).

${ }^{42}$ M. T. De Araujo, S. S. Vianna, and G. Grynberg, Opt. Commun. 80, 79 (1990).

${ }^{43}$ A. Yariv, Optical Electronics, 3-rd ed., Holt, Rinehart and Winston, New York (1985), pp. 28-34.

${ }^{44}$ R. W. Boyd, Nonlinear Optics, Academic, Boston (1992), pp. 245-252, 337, 343-347.

${ }^{45} \mathrm{P}$. Yeh, Introduction to Photorefractive Nonlinear Optics, Willey, New York (1993), pp. 211-220.

${ }^{46}$ V. G. Koptev, A. M. Lazaruk, I. P. Petrovich, and A. S. Rubanov, JETP Lett. 28, 434 (1978).

${ }^{47}$ G. S. He, Y. Cui, M. Yoshida, and P. N. Prasad, Opt. Lett. 22, 10 (1997).

${ }^{48}$ G. S. He and P. N. Prasad, J. Opt. Soc. Am. B 15, 1078 (1998).

${ }^{49}$ G. S. He, N. Cheng, P. N. Prasad, D. Liu, and S. H. Liu, J. Opt. Soc. Am. B 15, 1086 (1998).

${ }^{50}$ G. S. He and P. N. Prasad, IEEE J. Quantum Electron. 34, 473 (1998).

Published in English in the original Russian journal. Reproduced here with stylistic changes by the Translation Editor. 